\title{
A six-gene support vector machine classifier contributes to the diagnosis of pediatric septic shock
}

\author{
GUOLI LONG and CHEN YANG \\ Department of The Intensive Care Unit, Eastern Hospital, Sichuan Academy of Medical Sciences \\ and Sichuan Provincial People's Hospital, Chengdu, Sichuan 610101, P.R. China
}

Received April 21, 2019; Accepted November 12, 2019

DOI: $10.3892 / \mathrm{mmr} .2020 .10959$

\begin{abstract}
Septic shock is induced by an uncontrolled inflammatory immune response to pathogens and the survival rate of patients with pediatric septic shock (PSS) is particularly low, with a mortality rate of $25-50 \%$. The present study explored the mechanisms of PSS using four microarray datasets (GSE26378, GSE26440, GSE13904 and GSE4607) that were obtained from the Gene Expression Omnibus database. Based on the MetaDE package, the consistently differentially expressed genes (DEGs) in the four datasets were screened. Using the WGCNA package, the disease-associated modules and genes were identified. Subsequently, the optimal feature genes were further selected using the caret package. Finally, a support vector machine (SVM) classifier based on the optimal feature genes was built using the e1071 package. Initially, there were 2,699 consistent DEGs across the four datasets. From the 10 significantly stable modules across the datasets, four stable modules (including the magenta, purple, turquoise and yellow modules), in which the consistent DEGs were significantly enriched $(\mathrm{P}<0.05)$, were further screened. Subsequently, six optimal feature genes (including cysteine rich transmembrane module containing 1, S100 calcium binding protein A9, solute carrier family 2 member 14 , stomatin, uridine phosphorylase 1 and utrophin) were selected from the genes in the four stable modules. Additionally, an effective SVM classifier was constructed based on the six optimal genes. The SVM classifier based on the six optimal genes has the potential to be applied for PSS diagnosis. This may improve the accuracy of early PSS diagnosis and suggest possible molecular targets for interventions.
\end{abstract}

Correspondence to: Dr Chen Yang, Department of The Intensive Care Unit, Eastern Hospital, Sichuan Academy of Medical Sciences and Sichuan Provincial People's Hospital, 585 Honghe North Road, Longquanyi, Chengdu, Sichuan 610101, P.R. China

E-mail: yangchen028@sina.com

Key words: pediatric septic shock, meta-analysis, differentially expressed genes, weighted gene co-expression network analysis, support vector machine classifier

\section{Introduction}

Septic shock (SS) is caused by an uncontrolled inflammatory immune response to pathogens (for example, bacteria, fungi, parasites and viruses) and occurs when sepsis results in dangerous hypotension and abnormal cellular metabolism (1). SS can lead to multiple organ failure and death, and in 2018 the mortality rate of SS was $25-50 \%$ (2). Innate and adaptive immune responses of hosts of different ages have various effects against sepsis $(3,4)$, with the survival rate of pediatric septic shock (PSS) being particularly low compared with other age groups (5). PSS is one of the main causes of mortality in critically ill children in pediatric intensive care units worldwide (6). Adult and pediatric septic shock differ in terms of pathophysiology, clinical presentation and therapeutic approaches (7). Hyperdynamic shock syndrome or warm shock occurs in $\sim 90 \%$ of adult patients, while severe hypovolemia often characterizes PSS (7). Activated protein C is the preferred treatment for adult patients, whereas plasma exchange is preferred for the treatment of pediatric patients with thrombocytopenia-associated multiple organ failure (7). The therapy regimens widely used in adults with SS have been shown to have little therapeutic effect on children (8). Therefore, investigation into the molecular mechanisms of PSS for the diagnosis and therapeutic management of patients with PSS is required.

Nuclear factor erythroid 2-related factor 2 (Nrf2)-linked genes are dysregulated in PSS and may affect fatty acid metabolism, peroxisome proliferator-activated receptors (PPARs) and retinoic acid receptor- $\alpha$ families, which are related to intermediary metabolism and oxidative stress in PSS (9). A recent study revealed a list of differentially expressed genes (DEGs) that are important for SS diagnosis and are implicated in the immune response, chemokine-mediated signaling, neutrophil chemotaxis and chemokine activity (10). Olfactomedin- 4 controls sepsis heterogeneity and may be a biomarker of a pathogenic neutrophil subset associated with organ failure and mortality caused by PSS (11). Moreover, there is evidence that serum propionic acid has diagnostic value for septic shock (12). Despite the aforementioned findings, the mechanisms contributing to PSS are largely unknown.

Support vector machine (SVM) classifiers are gaining significance as a robust classification tool in cancer 
genomics (13) and have been used for the diagnosis of various diseases, including chronic kidney disease (14) and acute coronary syndrome (15). In the present study, it was hypothesized that an SVM classifier based on optimal feature genes of PSS could facilitate the diagnosis of the disease. The present study aimed to accurately identify the key genes in PSS from DEGs between PSS and control samples, and constructed a SVM classifier for distinguishing patients with PSS from normal controls. The results from the present study could aid the development of appropriate treatment strategies for PSS.

\section{Materials and methods}

Data source and pre-processing. Using the Gene Expression Omnibus (GEO; www.ncbi.nlm.nih.gov/geo) database, 'septic shock' and 'pediatric' were used as keywords to search for relevant datasets. The criteria for eligible datasets were as follows: i) Included gene expression data of blood samples; ii) age information was available to ensure that the subjects were children; iii) there were both PSS samples and normal control samples; and iv) the total number of samples was $\geq 100$ and the number of samples that could be used for analysis was $\geq 50$. Finally, four microarray datasets based on the GPL570 (HG-U133_Plus_2) Affymetrix Human Genome U133 Plus 2.0 Array, including GSE26378 (9), GSE26440 (16), GSE13904 (17) and GSE4607 (18), were selected (Table I). The GSE13904 and GSE4607 datasets contained other samples, therefore, only the control and septic shock samples were extracted for analysis in the present study.

For pre-processing the raw data in the four datasets, data formats were converted using the oligo package (19) (version 1.40.2; www. bioconductor.org/packages/oligo. html) in $\mathrm{R}$ and normalization of gene expression values was performed using the unit-scale normalization algorithm (20).

Meta-analysis to identify the consistent DEGs in the four datasets. The four datasets included in the present study involved experimental tests of samples from different patients and thus they may have displayed different degrees of bias. To resolve this issue, the MetaQC package (21) (version 0.1.13; www.cran.r-project.org/web/packages/MetaQC/index.html) in $\mathrm{R}$ was adopted to perform quality control on the datasets. The quality control standards in the MetaQC package contained accuracy quality control (AQC) $g$ and AQCp, external quality control, consistency quality control (CQC) g and CQCp and internal quality control. Combined with the two-dimensional diagram of the principal component analysis (PCA) and the standardized mean rank, these datasets were further assessed and screened.

Following the quality control analysis, DEGs between PSS and normal control samples were analyzed using the MetaDE. ES method in the MetaDE package (22) (version 1.0.5; www. cran.r-project.org/web/packages/MetaDE). To ensure that gene expression was consistent across the four datasets, homogeneity test parameters were set as $\operatorname{tau}^{2}=0$ and Qpval $>0.05$. A false discovery rate $(\mathrm{FDR})<0.05$ was selected as the significance threshold for screening of the DEGs. Moreover, the genes with $\log _{2}$ fold-change $(\mathrm{FC})>0.5$ in at least one of the datasets were used for analysis.
Weighted gene co-expression network analysis (WGCNA). WGCNA is an algorithm based on high-throughput expression data, which is utilized for the construction of a co-expression network (23). In WGCNA, the GSE26440 dataset, which contained a relatively large number of samples, was taken as the main analysis dataset and the other three datasets were considered as the secondary analysis datasets. Using the WGCNA package (23) (version 1.61; www.cran.r-project. org/web/packages/WGCNA/) in R, all genes in the GSE26440 dataset were analyzed and screened for disease-associated modules and genes. The requirements were: Number of module genes $\geq 80$, cutHeight $=0.995$ and $\mathrm{P}<0.05$. Combined with the three secondary analysis datasets, significantly stable modules across the four datasets were screened. Module preservation across the four datasets was analyzed using the module preservation function of the WGCNA package. Using clinical information of the samples in the GSE26440 dataset, the correlations between each significantly stable module and clinical information were calculated using the WGCNA cor function (version 1.68; 127.0.0.1:13239/library/WGCNA/html/cor. html) and WGCNA corPvalueStudent function (version 1.68; 127.0.0.1:13239/library/WGCNA/html/corPvalueStudent.html).

The consistent DEGs were mapped into the significantly stable modules. Significant enrichment parameters of target genes in the modules were calculated using the hypergeometric algorithm (24): $\mathrm{f}(\mathrm{k}, \mathrm{N}, \mathrm{M}, \mathrm{n})=\mathrm{C}(\mathrm{k}, \mathrm{M}) \mathrm{xC}(\mathrm{n}-\mathrm{k}, \mathrm{N}-\mathrm{M}) / \mathrm{C}(\mathrm{n}, \mathrm{N})$, where $\mathrm{N}$ stands for the number of total genes participating in WGCNA network analysis, $M$ stands for the number of genes included in each module, $\mathrm{n}$ stands for the number of genes identified by the MetaDE method and $\mathrm{k}$ stands for the number of DEGs mapped to a module.

The thresholds of significant enrichment distribution were selected as $\mathrm{P}<0.05$ and fold enrichment $>1$. For the consistent DEGs included in the significantly stable modules, Gene Ontology (GO; www.geneontology.org) and Kyoto Encyclopedia of Genes and Genomes (KEGG; www.genome. $\mathrm{jp} / \mathrm{kegg}$ ) enrichment analyses were conducted based on the Database for Annotation, Visualization and Integrated Discovery tool (25) (version 6.8; ww.david.ncifcrf.gov). $\mathrm{P}<0.05$ was selected as the screening threshold.

Selection of the optimal gene combination and construction of the SVM classifier. In order to further narrow down the range of SS-related genes and accurately identify important feature genes, the GSE26440 dataset was used as the training dataset and the other three datasets were taken as the validation datasets to optimize the previously identified DEGs. Recursive feature elimination (RFE) is an integrated machine learning method, which regards the selection of subsets as an optimization problem and evaluates gene combinations (26). From the training dataset GSE26440, the combination of optimal feature genes were selected using the RFE method in the R caret package (27) (version 6.0-76; www.cran.r-project. org/web/packages/caret). In 10-fold cross validation, the gene combination with the highest accuracy and the lowest Root Mean Square Error (RMSE) was selected as the optimal gene combination.

SVM is a supervised classification algorithm for machine learning, which uses the eigenvalues of features in each sample to discriminate sample types and estimate the 
Table I. Information of the four microarray datasets.

Dataset

Platform

Samples (n) Control $\begin{aligned} & \text { Septic } \\ & \text { shock }\end{aligned}$

\begin{tabular}{lllll}
\hline GSE26378 & GPL570 (HG-U133_Plus_2) Affymetrix Human Genome U133 Plus 2.0 Array & 103 & 21 & 82 \\
GSE26440 & GPL570 (HG-U133_Plus_2) Affymetrix Human Genome U133 Plus 2.0 Array & 130 & 32 & 98 \\
GSE13904 $^{\text {a }}$ & GPL570 (HG-U133_Plus_2) Affymetrix Human Genome U133 Plus 2.0 Array & 227 & 18 & 67 \\
GSE4607 $^{\mathrm{a}}$ & GPL570 (HG-U133_Plus_2) Affymetrix Human Genome U133 Plus 2.0 Array & 123 & 15 & 42
\end{tabular}

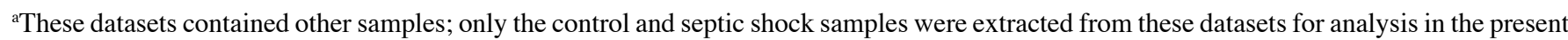
study.

Table II. Results of quality control analysis of the four microarray datasets.

\begin{tabular}{lccrrrrr}
\hline Dataset & IQC & EQC & CQCg & CQCp & AQCg & AQCp & SMR \\
\hline GSE26378 & 5.350 & 4.453 & 237.678 & 107.220 & 41.268 & 114.658 & 2.839 \\
GSE26440 & 6.089 & 3.784 & 246.767 & 102.538 & 33.131 & 94.224 & 3.561 \\
GSE13904 & 6.448 & 4.579 & 74.753 & 76.039 & 37.205 & 106.445 & 4.101 \\
GSE4607 & 6.649 & 3.141 & 127.552 & 114.078 & 24.009 & 74.664 & 4.202 \\
\hline
\end{tabular}

IQC, internal quality control; EQC, external quality control; CQCg, consistency quality control gene; CQCp, consistency quality control pathway; AQCg, accuracy quality control gene; AQCp, accuracy quality control pathway; SMR, standardized mean rank.

A

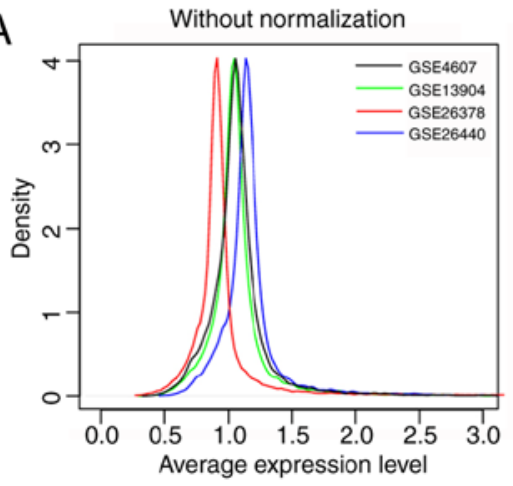

B

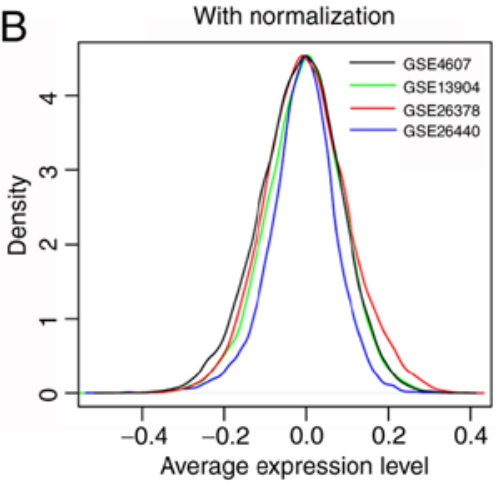

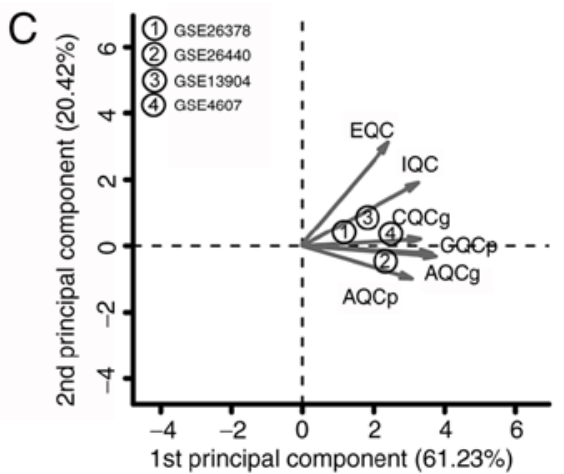

Figure 1. Distribution curves and a two-dimensional diagram of the PCA for the four datasets. Distribution curves (A) before and (B) after normalization. (C) Two-dimensional diagram of the PCA. Distribution curves for the GSE4607, GSE13904, GSE26378 and GSE26440 datasets are represented in black, green, red and blue, respectively. PCA, principal component analysis; IQC, internal quality control; EQC, external quality control; CQCg, consistency quality control gene; CQCp, consistency quality control pathway; AQCg, accuracy quality control gene; AQCp, accuracy quality control pathway.

probability that a sample belongs to a certain class (28). Using the SVM method in the e1071 package (28) (version 1.6-8; www.cran.r-project.org/web/packages/e1071) in $\mathrm{R}$, the SVM classifier (Core; Sigmoid Kernel; Cross; 100-fold Cross validation) based on the optimal gene combination was built. Based on the pROC package (29) (version 1.12.1; www.cran.r-project.org/web/packages/pROC/index.html) in $\mathrm{R}$, the area under the receiver operating characteristic (AUROC) was used to evaluate the efficiency of the SVM classifier in the training and validation datasets. Values of AUROC were distributed between 0.5 and 1 , and the closer the AUROC value was to 1 , the higher the efficiency of the classifier.

\section{Results}

Meta-analysis to identify the consistent DEGs across four datasets. The expression data in the four datasets were standardized; the curves before and after standardization are shown in Fig. 1A and B, respectively. After standardization, the gene expression levels of each dataset were distributed between -1 and 1 , and the peak expression level was $\sim 0$, which was uniform (Fig. 1B). The results of the quality control analysis (Table II) and the PCA diagram (Fig. 1C) suggested that the distributions of the four datasets were balanced and all indexes met the quality test standards. Therefore, all datasets could be included in the subsequent analyses. 


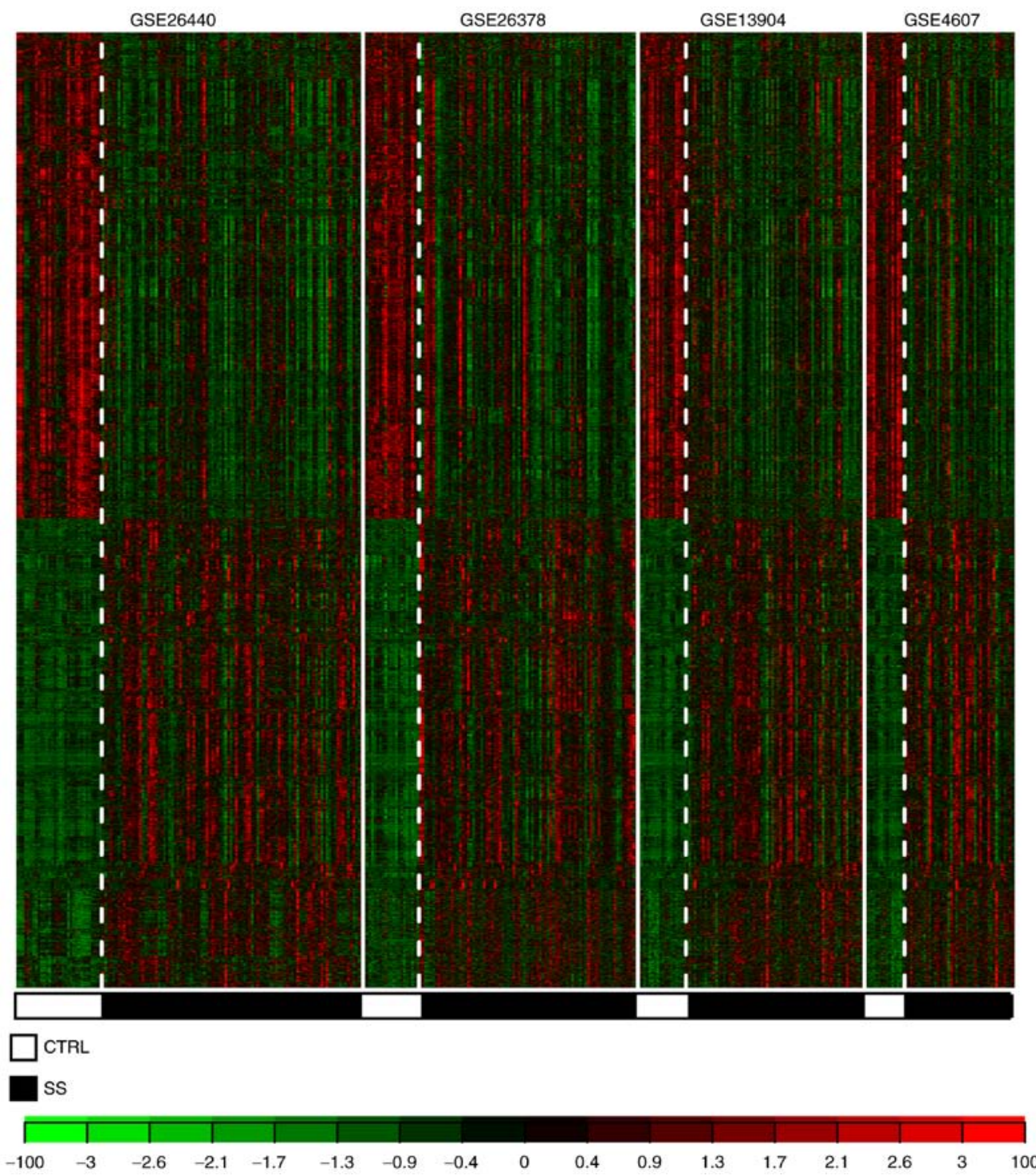

Figure 2. Heat map of the 2,699 consistent differentially expressed genes. Black and white sample strips represent pediatric septic shock and control samples, respectively. Red, green and black dots represent upregulated, downregulated and unchanged genes, respectively. CTRL, control; SS, septic shock.

A

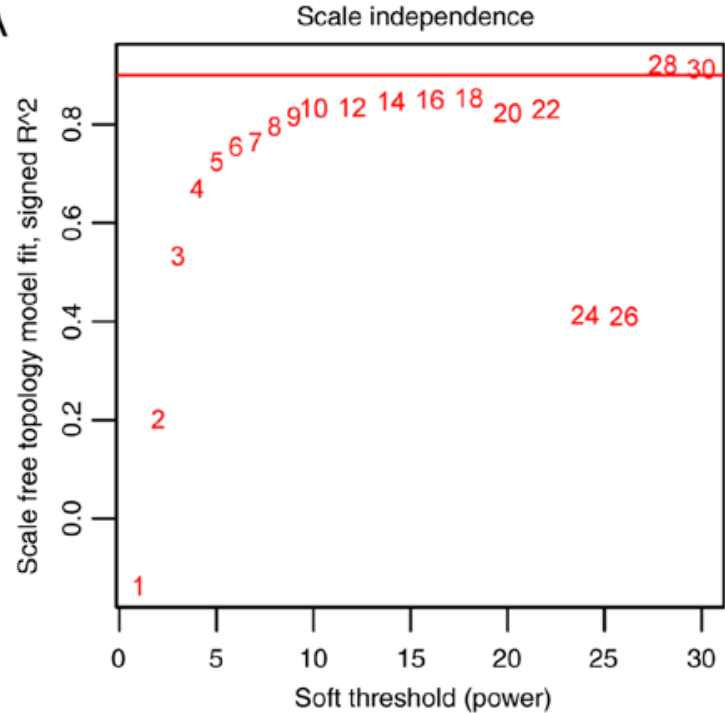

B

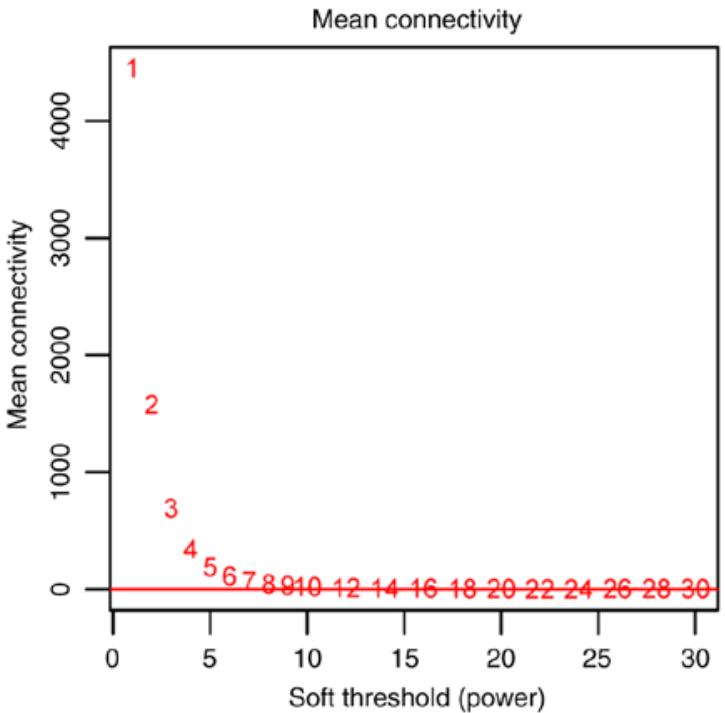

Figure 3. Selection graph of the weighting parameter 'power' and schematic diagram of mean connectivity. (A) Selection graph of the weighting parameter 'power'. The red line is the standard line when the square of the correlation coefficient reaches 0.9 . (B) Schematic diagram of the mean RNA connectivity under different power parameters; when the power $=28$, the mean connectivity is 1 . 
Table III. Preservation and enrichment information of the 11 modules and the module genes.

\begin{tabular}{|c|c|c|c|c|c|c|c|}
\hline \multirow[b]{2}{*}{ Module ID } & \multirow[b]{2}{*}{ Color } & \multirow[b]{2}{*}{ Module size } & \multicolumn{2}{|c|}{$\begin{array}{l}\text { Preservation } \\
\text { information }\end{array}$} & \multirow[b]{2}{*}{ DEGs (n) } & \multicolumn{2}{|c|}{ Enrichment information } \\
\hline & & & Z-score & P-value & & Enrichment fold $(95 \% \mathrm{CI})$ & Phyper \\
\hline Module 1 & Black & 95 & 11.823 & $1.78 \times 10^{-4}$ & 0 & - & - \\
\hline Module 2 & Blue & 139 & 9.292 & $1.18 \times 10^{-2}$ & 1 & $0.157(0.004-0.898)$ & $3.13 \times 10^{-2}$ \\
\hline Module 3 & Brown & 137 & 20.037 & $1.80 \times 10^{-3}$ & 1 & $0.159(0.004-0.912)$ & $3.09 \times 10^{-2}$ \\
\hline Module 4 & Green & 115 & 21.756 & $1.57 \times 10^{-10}$ & 3 & $0.569(0.115-1.727)$ & $4.90 \times 10^{-1}$ \\
\hline Module 5 & Grey & 3,907 & 8.083 & $1.00 \times 10^{-200}$ & 55 & $0.307(0.224-0.415)$ & $2.20 \times 10^{-16}$ \\
\hline Module 6 & Magenta & 85 & 14.990 & $3.17 \times 10^{-6}$ & 12 & $3.079(1.509-5.766)$ & $1.30 \times 10^{-3}$ \\
\hline Module 7 & Pink & 91 & 20.320 & $1.10 \times 10^{-10}$ & 2 & $0.479(0.057-1.803)$ & $4.40 \times 10^{-1}$ \\
\hline Module 8 & Purple & 82 & 22.134 & $6.00 \times 10^{-4}$ & 26 & $6.915(4.186-11.103)$ & $2.67 \times 10^{-12}$ \\
\hline Module 9 & Red & 105 & 21.649 & $1.57 \times 10^{-6}$ & 8 & $1.662(0.691-3.452)$ & $1.64 \times 10^{-1}$ \\
\hline Module 10 & Turquoise & 288 & 36.143 & $7.00 \times 10^{-38}$ & 67 & $5.074(3.714-6.868)$ & $2.20 \times 10^{-16}$ \\
\hline Module 11 & Yellow & 128 & 23.321 & $3.20 \times 10^{-34}$ & 62 & $10.559(7.458-14.849)$ & $2.20 \times 10^{-16}$ \\
\hline
\end{tabular}

The higher the stability parameter (Z-score), the higher the stability of the module. In general, $5<\mathrm{Z}<10$ indicates stability and $\mathrm{Z}>10$ indicates high stability. Phyper, P-value calculated by the hypergeometric algorithm; DEGs, differentially expressed genes; CI, confidence interval; - , not applicable.

A

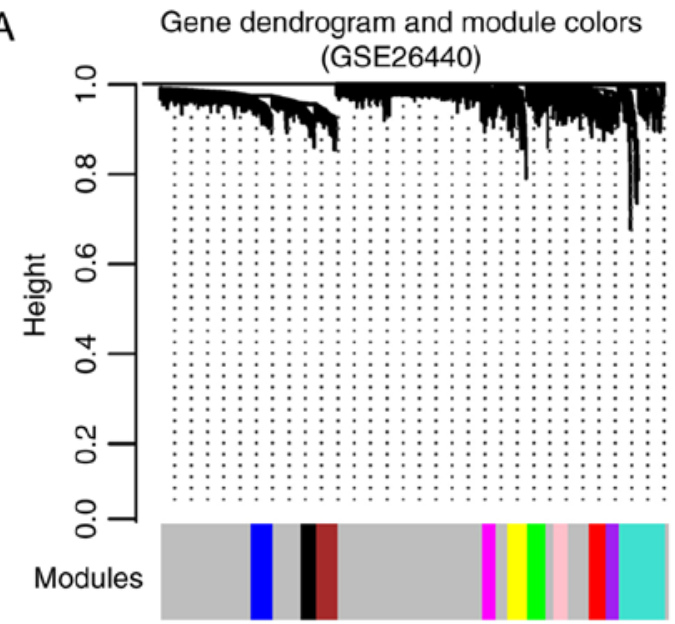

C

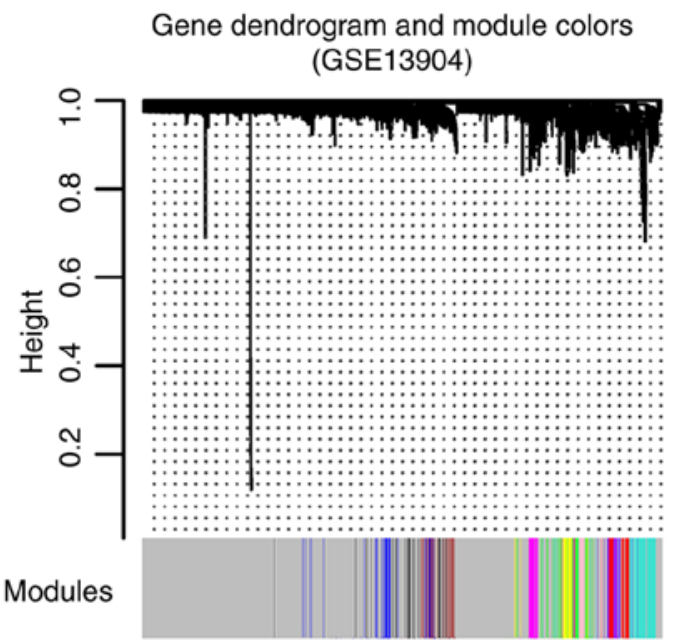

B Gene dendrogram and module colors (GSE26378)

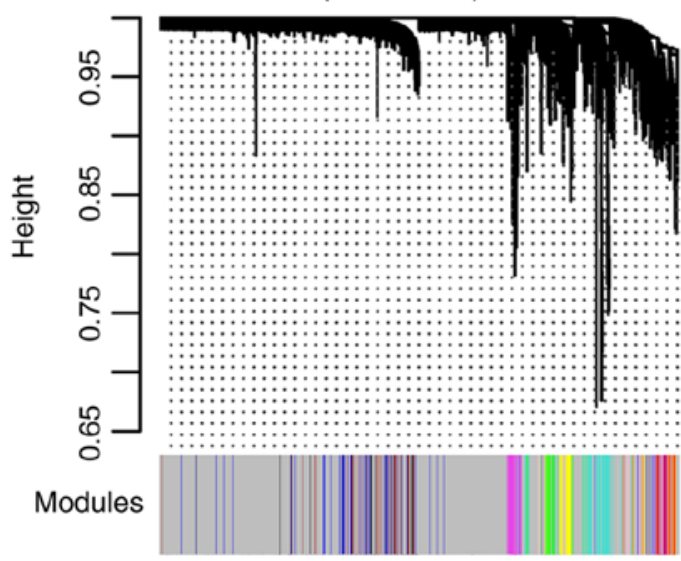

$\mathrm{D}$

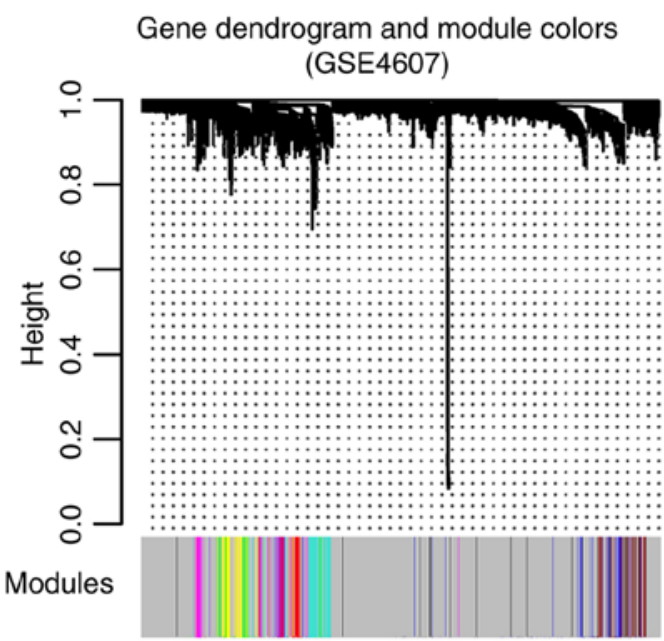

Figure 4. Tree diagrams for the partitioning modules. Tree diagram for (A) GSE26440, (B) GSE26378, (C) GSE13904 and (D) GSE4607. Each color represents a different module. 
A

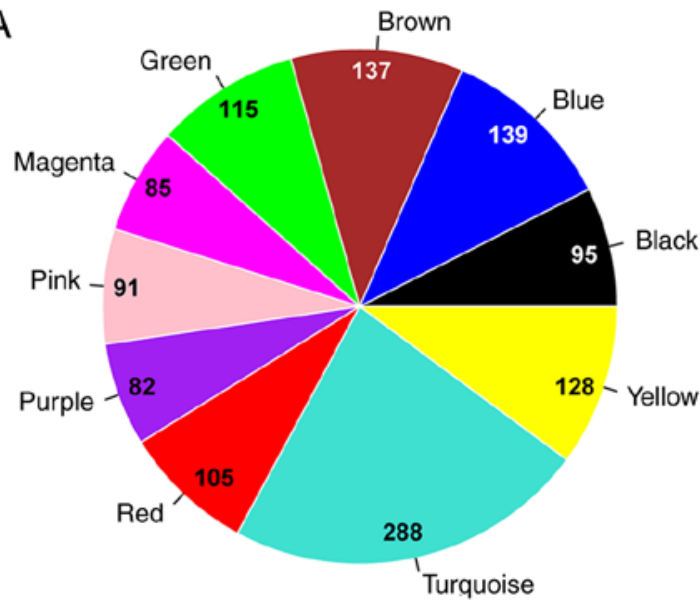

B

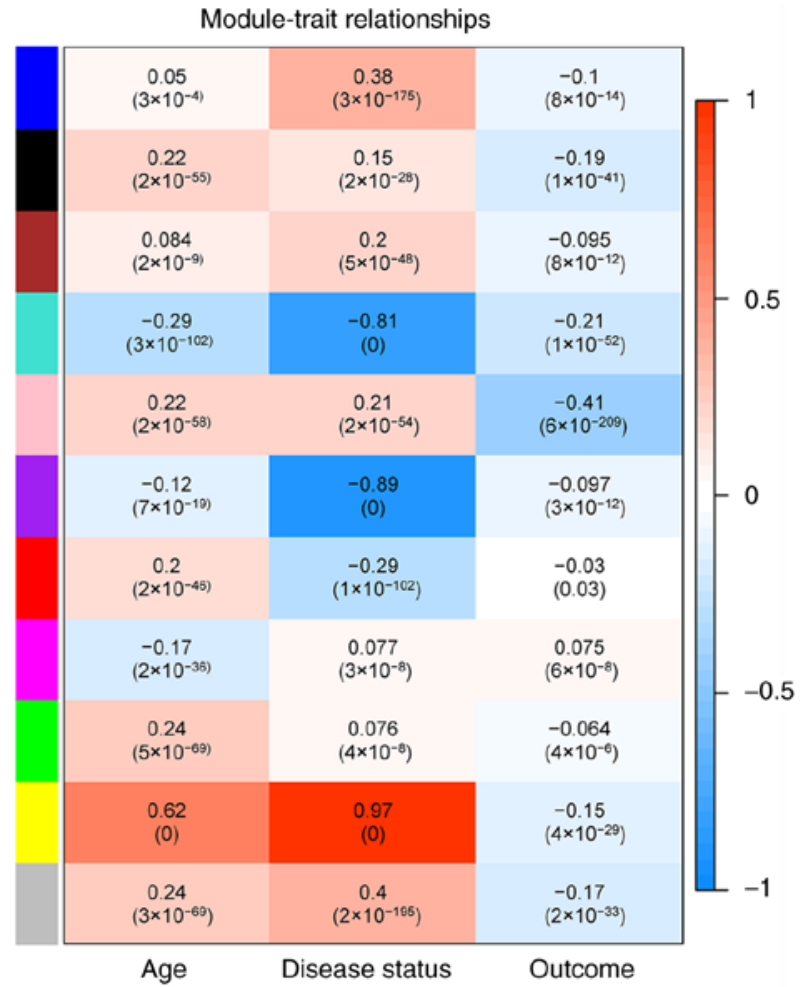

Figure 5. Pie chart and clinical information correlation heat map of significantly stable modules. (A) Pie chart displaying the number of genes contained in each of the 10 significantly stable modules. (B) Correlation heat map between each significantly stable module and the clinical information of patients, including age, disease status and outcome. The data are presented as the correlation coefficient (P-value). Correlations were calculated using WGCNA cor and corPvalueStudent packages. The color from blue to orange indicates the correlation from -1 to 1 , respectively.

Through comprehensive analysis using the MetaDE package, the P, FDR, tau ${ }^{2}, \mathrm{Q}$ pval and Q values for each gene, as well as the $\log _{2} \mathrm{FC}$ value in each dataset, were calculated. According to the pre-set thresholds, a total of 2,699 consistent DEGs were identified from the four datasets. The heat map suggested that the differential expression patterns of the 2,699 DEGs were consistent in the four datasets (Fig. 2).

WGCNA. To ensure that the gene expression levels in each dataset were comparable, all gene expression values in the four datasets were analyzed for expression level consistency. The expression level and connection level correlations between all combinations of pairs within the four datasets were positive and the $\mathrm{P}$-values were significant $\left(\mathrm{P}<1 \times 10^{-200}\right)$, indicating that the datasets were comparable (Fig. S1).

WGCNA needed to satisfy the pre-condition of scale-free network distribution. Therefore, the value of the weighting parameter 'power' was firstly explored. Based on the GSE26440 dataset, the square values of the correlation coefficients between $\log (\mathrm{k})$ and $\log [\mathrm{p}(\mathrm{k})]$ corresponding to different power values were calculated. Finally, the 'power' value was selected to be 28 when the square value of the correlation coefficient reached 0.9 (Fig. 3A). Under the parameter of 'power' $=28$, the mean gene connectivity was statistically analyzed. The mean connectivity was 1 , which confirmed the small-world property of the scale-free connection network (Fig. 3B).

After the co-expression network was constructed using the GSE26440 dataset as the main analysis dataset, a total of 11 modules were selected (Fig. 4A). The other three datasets including GSE26378 (Fig. 4B), GSE13904 (Fig. 4C) and GSE4607 (Fig. 4D) were also constructed with module partition. Meanwhile, module stability was evaluated and 10 significantly stable modules across the datasets were obtained (Table III). The number of genes present in each significantly stable module are shown in Fig. 5A. Using clinical information of the samples in the GSE26440 dataset, the correlations between each significantly stable module and clinical information were calculated using the WGCNA cor and corPvalueStudent packages (Fig. 5B).

The consistent DEGs were compared to the genes within the significantly stable modules, resulting in the identification of 237 overlapping genes. Enrichment and distribution situations of the overlapping genes in each significantly stable module are presented in Table III. The consistent DEGs were significantly enriched in four stable modules, including the magenta, purple, turquoise, and yellow modules, which contained 12 , 26, 67 and 62 genes, respectively $(\mathrm{P}<0.05)$. Afterwards, enrichment analysis for the genes involved in the four stable modules was performed and $18 \mathrm{GO}$ biological process terms, including 'translational elongation' $\left(\mathrm{P}=6.45 \times 10^{-16}\right)$ and 'translation' $\left(\mathrm{P}=2.16 \times 10^{-13}\right)$, as well as nine KEGG pathways, including 'ribosome' $\left(\mathrm{P}=1.55 \times 10^{-14}\right)$ and ' $\mathrm{Fc}$ gamma R-mediated phagocytosis' $\left(\mathrm{P}=1.89 \times 10^{-3}\right)$ were acquired (Table IV).

Selection of the optimal gene combination and construction of SVM classifier. With the GSE26440 dataset as the training dataset, the optimal feature genes were further identified from the genes involved in the four stable modules. Under the optimal parameters (min RMSE $=0.0849$ and $\max$ accuracy $=0.9262$ ), six optimal feature genes [cysteine rich 
Table IV. GO biological process terms and KEGG pathways enriched for the genes involved in the four stable modules.

A, GO analysis

\begin{tabular}{lrr}
\hline Biological process term & Count & P-value \\
\hline GO:0006414: Translational elongation & 17 & $6.45 \times 10^{-16}$ \\
GO:0006412: Translation & 23 & $2.16 \times 10^{-13}$ \\
GO:0030029: Actin filament-based process & 10 & $3.51 \times 10^{-4}$ \\
GO:0030036: Actin cytoskeleton organization & 9 & $1.06 \times 10^{-3}$ \\
GO:0046907: Intracellular transport & 15 & $2.55 \times 10^{-3}$ \\
GO:0042254: Ribosome biogenesis & 6 & $5.09 \times 10^{-3}$ \\
GO:0022613: Ribonucleoprotein complex biogenesis & 7 & $6.01 \times 10^{-3}$ \\
GO:0006413: Translational initiation & 4 & $7.88 \times 10^{-3}$ \\
GO:0006928: Cell motion & 11 & 12 \\
GO:0016192: Vesicle-mediated transport & 9 & $1.13 \times 10^{-2}$ \\
GO:0034621: Cellular macromolecular complex subunit organization & 10 & $1.58 \times 10^{-2}$ \\
GO:0007010: Cytoskeleton organization & 9 & $1.62 \times 10^{-2}$ \\
GO:0006886: Intracellular protein transport & 9 & $1.80 \times 10^{-2}$ \\
GO:0034613: Cellular protein localization & $9.08 \times 10^{-2}$ \\
GO:0070727: Cellular macromolecule localization & 9 & $3.39 \times 10^{-2}$ \\
GO:0015031: Protein transport & 13 & $3.52 \times 10^{-2}$ \\
GO:0001667: Ameboidal cell migration & 3 & $4.44 \times 10^{-2}$ \\
GO:0045184: Establishment of protein localization & 13 & $4.44 \times 10^{-2}$ \\
\end{tabular}

B, KEGG analysis

\begin{tabular}{lrr}
\hline Pathway & Count & P-value \\
\hline hsa03010: Ribosome & 15 & $1.55 \times 10^{-14}$ \\
hsa04666: Fc gamma R-mediated phagocytosis & 5 & $1.89 \times 10^{-3}$ \\
hsa04810: Regulation of actin cytoskeleton & 6 & $8.13 \times 10^{-3}$ \\
hsa05110: Vibrio cholerae infection & 3 & $1.22 \times 10^{-2}$ \\
hsa04062: Chemokine signaling pathway & 4 & $3.30 \times 10^{-2}$ \\
hsa04130: SNARE interactions in vesicular transport & 2 & $3.40 \times 10^{-2}$ \\
hsa04670: Leukocyte transendothelial migration & 3 & $3.66 \times 10^{-2}$ \\
hsa04360: Axon guidance & 3 & $4.09 \times 10^{-2}$ \\
hsa00190: Oxidative phosphorylation & 3 & $4.13 \times 10^{-2}$ \\
\hline
\end{tabular}

GO, Gene Ontology; KEGG, Kyoto Encyclopedia of Genes and Genomes.

Table V. Indexes for assessing the efficiency of the support vector machine classifier in the training and validation datasets.

\begin{tabular}{|c|c|c|c|c|c|c|}
\hline Dataset & Precision rate & Sensitivity & Specificity & PPV & NPV & AUROC \\
\hline GSE26440 & 0.977 & 0.989 & 0.939 & 0.979 & 0.969 & 0.994 \\
\hline GSE26378 & 0.961 & 1.000 & 0.840 & 0.951 & 1.000 & 0.990 \\
\hline GSE13904 & 0.976 & 1.000 & 0.900 & 0.970 & 1.000 & 0.983 \\
\hline GSE4607 & 0.947 & 0.976 & 0.875 & 0.952 & 0.933 & 0.951 \\
\hline
\end{tabular}

Precision rate was calculated as follows: True positive/(true positive + false positive). PPV, positive predictive value; NPV, negative predictive value; AUROC, area under receiver operating characteristic.

transmembrane module containing 1 (CYSTM1), S100 calcium binding protein A9 (S100A9), solute carrier family 2 member
14 (SLC2A14), stomatin (STOM), uridine phosphorylase 1 (UPP1) and utrophin (UTRN)] were selected. 


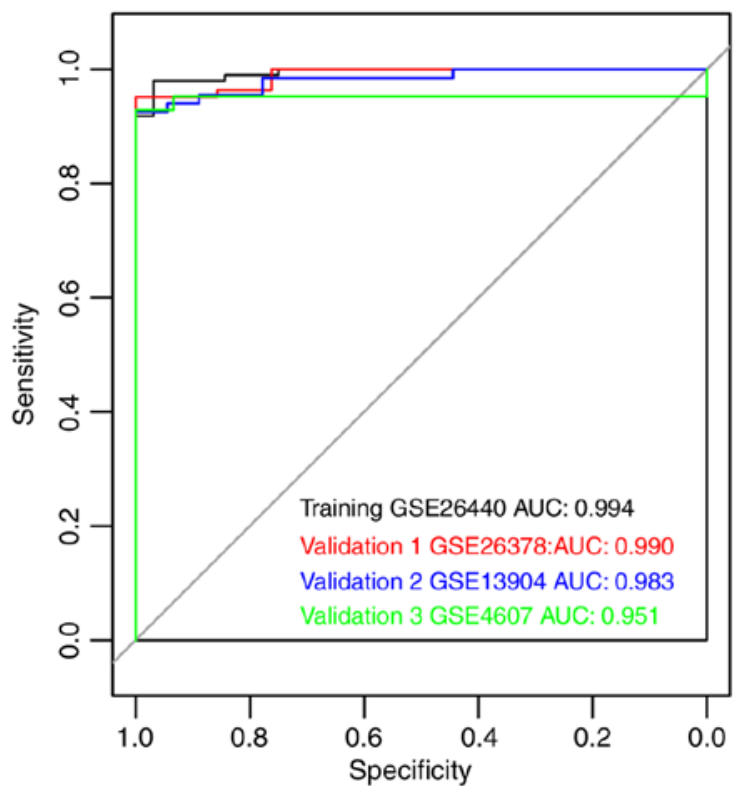

Figure 6. ROC curves based on the six optimal feature genes. ROC curves for GSE26440, GSE26378, GSE13904 and GSE4607 are displayed in black, red, blue and green, respectively. ROC, receiver operating characteristic.

A

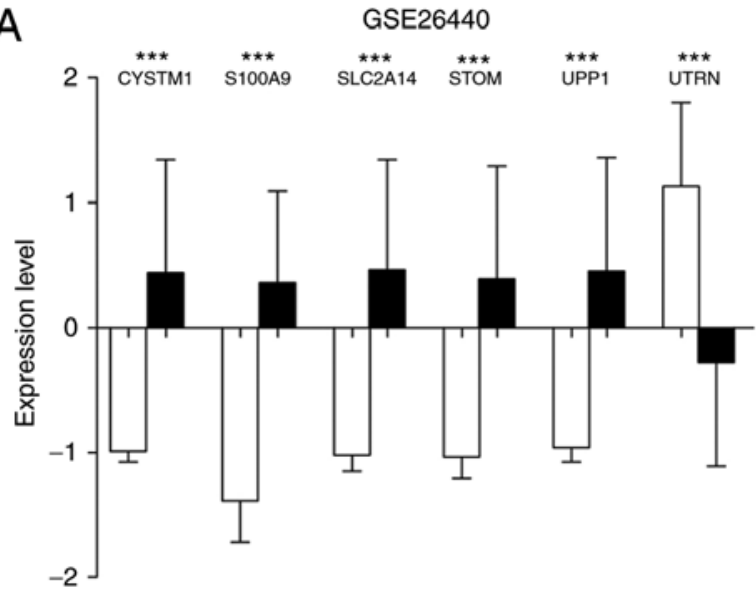

$\mathrm{C}$

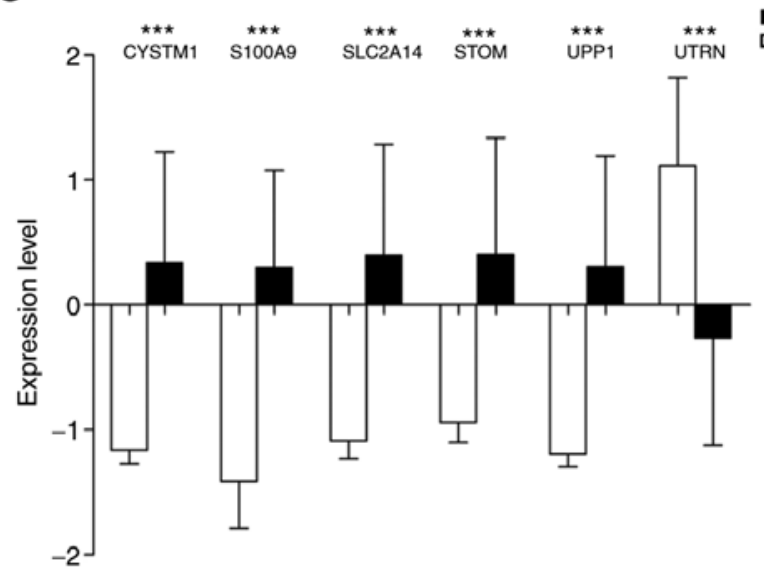

B

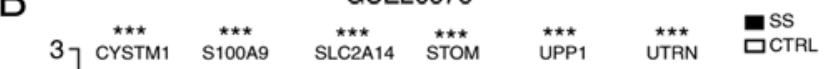

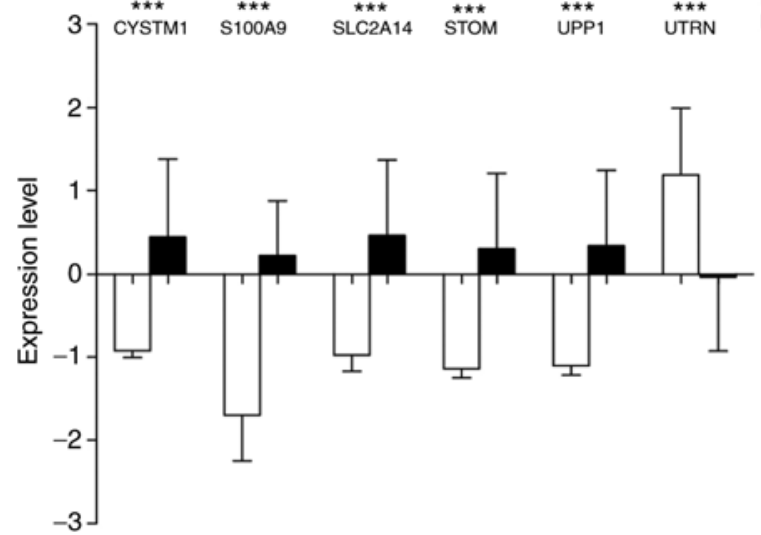

D GSE4607

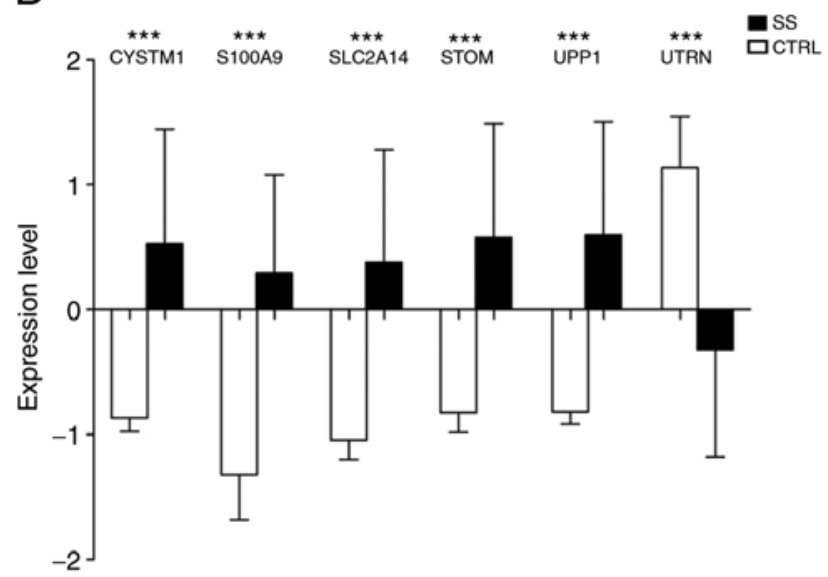

Figure 7. Expression of the six optimal genes in the four datasets. Column chart for (A) GSE26440, (B) GSE26378, (C) GSE13904 and (D) GSE4607. ${ }^{* * *} \mathrm{P}<0.005$ vs. the control group. SS, septic shock; CTRL, control; CYSTM1, cysteine rich transmembrane module containing 1; S100A9, S100 calcium binding protein A9; SLC2A14, solute carrier family 2 member 14; STOM, stomatin; UPP1, uridine phosphorylase 1; UTRN, utrophin.

Based on the six optimal feature genes, an SVM classifier was constructed within the GSE26440 dataset. Subsequently, the efficiency of the SVM classifier in the training and validation datasets was assessed. All the precision rates, calculated 
as true positive/(true positive + false positive), (Table V) and AUROC values (Fig. 6) were $>0.9$, suggested that the SVM classifier could accurately discriminate PSS samples from control samples. In addition, the expression of the six optimal genes in the four datasets was analyzed. The results suggested that the expression differences of the six optimal genes were consistent in the four datasets. The UTRN gene was significantly downregulated in the PSS samples $(\mathrm{P}<0.005)$, whereas the other five genes were significantly upregulated in the PSS samples $(\mathrm{P}<0.005$; Fig. 7).

\section{Discussion}

In the present study, 2,699 consistent DEGs were screened from four datasets and 10 significantly stable modules across the datasets were obtained, based on WGCNA. Subsequently, the consistent DEGs were found to be enriched in the four stable modules, including the magenta, purple, turquoise, and yellow modules, which contained 12, 26, 67 and 62 genes, respectively. Moreover, six optimal feature genes (CYSTM1, S100A9, SLC2A14, STOM, UPP1 and UTRN) were identified from the genes included in the four stable modules. Additionally, an effective SVM classifier based on the six optimal genes was constructed. The results from the ROC curve analysis showed that the SVM classifier had high sensitivity and specificity in discriminating patients with PSS from normal subjects. To the best of our knowledge, an SVM classifier for SS diagnosis has not been reported previously. Therefore, the SVM classifier of six optimal genes developed in the present study may aid in the early identification of patients with PSS in clinical practice.

S100A9, constitutively expressed in neutrophils, is a member of the alarmins family, and exhibits several immune functions, including immunological defense and homeostasis (30). Upregulation of S100A9 at the mRNA level in SS is related to the occurrence of hospital-acquired infections following SS and may contribute to the early identification of patients at high risk of infection (31). Moreover, S100A8/S100A9 alarmins compromise the suppression of the immune system by myeloid-derived suppressor cells, a specific inflammatory monocyte population, by repressing their expansion, thus preventing the development of SS in neonates (32). Endotoxin tolerance (ET) is a critical immune dysfunction related to SS (33). Elevated S100A8 and S100A9 expression has been shown to be induced in ex vivo models of ET, suggesting that these two genes may serve as promising biomarkers of ET and therefore providing valuable information for immunotherapy of patients with SS (34). The present study suggested that S100A9 is closely related to the development and diagnosis of PSS. CYSTM1 confers tolerance and stress responses to heavy metals (35) and is a novel biomarker in Huntington's disease (36). The role of CYSTM1 in PSS is not completely understood. The results of the present study indicated that CYSTM1 might have a role in the mechanisms of PSS by interacting with other genes in the stable modules.

Previous studies have revealed that genetic variations in SLC2A14 are involved in the development and progression of chronic diseases, including inflammatory bowel disease (IBD) and Alzheimer's disease (37-39). The facilitated glucose transporter 14 encoded by SLC2A14 promotes the development of IBD and may be applied for precision intervention of IBD (38). SLP-2 belongs to the stomatin protein family, plays a critical role in $\mathrm{T}$ cell activation and is a candidate target for immunomodulation (40). STOM expression is dysregulated between septic children and healthy controls and may be a diagnostic marker for pediatric sepsis (41). The present study revealed that SLC2A14 and STOM might be involved in the pathogenesis of PSS by mediating the inflammatory immune response.

UPP1 functions in the homeostatic regulation of intracellular uridine concentrations and the activation of fluoropyrimidine nucleoside chemotherapeutic agents (42). Uridine displays anti-inflammatory action during lung inflammation (43). UTRN upregulation is implicated in the immune reaction in Duchenne muscular dystrophy mouse models (44). UTRN upregulation induced by proinflammatory factor-associated post-transcriptional mechanisms exhibits an antidystrophic effect (45). Therefore, the present study suggested that UPP1 and UTRN might act in the development and progression of PSS by affecting inflammation and immune reactions as well.

To the best of our knowledge, the present study is the first to report the diagnostic value of the aforementioned six genes for PSS. The six genes may be useful biomarkers for the early detection of PSS. However, in the present study the genes were not confirmed experimentally, therefore, further investigation is required to validate the results of the present study.

In conclusion, 2,699 consistent DEGs from four GEO datasets were analyzed in the present study. Furthermore, a SVM classifier based on six optimal genes was constructed for the accurate diagnosis of PSS, which may assist in the early identification of PSS and provide useful guidance for clinical interventions.

\section{Acknowledgements}

Not applicable.

\section{Funding}

No funding was received.

\section{Availability of data and materials}

The datasets analyzed during the current study are available in the Gene Expression Omnibus database (www.ncbi.nlm.nih. gov/geo) with the following accession numbers: GSE26378, GSE26440, GSE13904 and GSE4607.

\section{Authors' contributions}

GL analyzed and interpreted the microarray datasets. CY designed the study and majorly contributed to writing the manuscript. All authors read and approved the final manuscript.

\section{Ethics approval and consent to participate}

Not applicable. 


\section{Patient consent for publication}

Not applicable.

\section{Competing interests}

The authors declare that they have no competing interests.

\section{References}

1. Rhee $\mathrm{C}$ and Klompas $\mathrm{M}$ : New sepsis and septic shock definitions: Clinical implications and controversies. Infect Dis Clin North Am 31: 397-413, 2017.

2. de Grooth HJ, Parienti JJ, Postema J, Loer SA, Oudemansvan Straaten HM and Girbes AR: Positive outcomes, mortality rates, and publication bias in septic shock trials. Intensive Care Med 44: 1584-1585, 2018

3. Checchia PA, Schierding W, Polpitiya A, Dixon D, Macmillan S Muenzer J, Stromberg P, Coopersmith CM, Buchman TG and Cobb JP: Myocardial transcriptional profiles in a murine mode of sepsis: Evidence for the importance of age. Pediatr Crit Care Med 9: 530-535, 2008.

4. Wynn J, Cornell TT, Wong HR, Shanley TP and Wheeler DS: The host response to sepsis and developmental impact. Pediatrics 125 : 1031-1041, 2010.

5. Watson RS and Carcillo JA: Scope and epidemiology of pediatric sepsis. Pediatr Crit Care Med 6 (3 Suppl): S3-S5, 2005.

6. Schlapbach LJ, Straney L, Alexander J, MacLaren G, Festa M, Schibler A and Slater A; ANZICS Paediatric Study Group: Mortality related to invasive infections, sepsis, and septic shock in critically ill children in Australia and New Zealand, 2002-13: A multicentre retrospective cohort study. Lancet Infect Dis 15 46-54, 2015.

7. Aneja RK and Carcillo JA: Differences between adult and pediatric septic shock. Minerva Anestesiol 77: 986-992, 2011.

8. Polat G, Ugan RA, Cadirci E and Halici Z: Sepsis and septic shock: Current treatment strategies and new approaches. Eurasian J Med 49: 53-58, 2017.

9. Grunwell JR, Weiss SL, Cvijanovich NZ, Allen GL, Thomas NJ, Freishtat RJ, Anas N, Meyer K, Checchia PA, Shanley TP, et al: Differential expression of the Nrf2-linked genes in pediatric septic shock. Crit Care 19: 327, 2015.

10. Mohammed A, Cui Y, Mas VR and Kamaleswaran R: Differential gene expression analysis reveals novel genes and pathways in pediatric septic shock patients. Sci Rep 9: 11270, 2019.

11. Alder MN, Opoka AM, Lahni P, Hildeman DA and Wong HR: Olfactomedin-4 is a candidate marker for a pathogenic neutrophil subset in septic shock. Crit Care Med 45: e426-e432, 2017.

12. Weng J, Wu H, Xu Z, Xi H, Chen C, Chen D, Gong Y, Hua Y and Wang Z: The role of propionic acid at diagnosis predicts mortality in patients with septic shock. J Crit Care 43: 95-101, 2018.

13. Huang S, Cai N, Pacheco PP, Narrandes S, Wang Y and Xu W: Applications of support vector machine (SVM) learning in cancer genomics. Cancer Genomics-Proteomics 15: 41-51, 2018.

14. Polat H, Danaei Mehr H and Cetin A: Diagnosis of chronic kidney disease based on support vector machine by feature selection methods. J Med Syst 41: 55, 2017

15. Berikol GB, Yildiz O and Özcan İT: Diagnosis of acute coronary syndrome with a support vector machine. J Med Syst 40: 84 2016.

16. Wong HR, Cvijanovich N, Lin R, Allen GL, Thomas NJ, Willson DF, Freishtat RJ, Anas N, Meyer K, Checchia PA, et al: Identification of pediatric septic shock subclasses based on genome-wide expression profiling. BMC Med 7: 34, 2009.

17. Wong HR, Cvijanovich N, Allen GL, Lin R, Anas N, Meyer K, Freishtat RJ, Monaco M, Odoms K, Sakthivel B, et al: Genomic expression profiling across the pediatric systemic inflammatory response syndrome, sepsis, and septic shock spectrum. Crit Care Med 37: 1558-1566, 2009.

18. Cvijanovich N, Shanley TP, Lin R, Allen GL, Thomas NJ, Checchia P, Anas N, Freishtat RJ, Monaco M, Odoms K, et al: Validating the genomic signature of pediatric septic shock. Physiol Genomics 34: 127-134, 2008.

19. Parrish RS and Spencer HJ III: Effect of Normalization on significance testing for oligonucleotide microarrays. J Biopharm Stat 14: 575-589, 2004.
20. Chaudhary K, Poirion OB, Lu L and Garmire LX: Deep Learning-based multi-omics integration robustly predicts survival in liver cancer. Clin Cancer Res 24: 1248-1259, 2018.

21. Zhou Q, Su X, Jing G and Ning K: Meta-QC-Chain: Comprehensive and fast quality control method for metagenomic data. Genomics Proteomics Bioinformatics 12: 52-56, 2014.

22. Chang LC, Lin HM, Sibille E and Tseng GC: Meta-analysis methods for combining multiple expression profiles: Comparisons, statistical characterization and an application guideline. BMC Bioinformatics 14: 368, 2013.

23. Li J, Zhou D, Qiu W, Shi Y, Yang JJ, Chen S, Wang Q and Pan H: Application of weighted gene co-expression network analysis for data from paired design. Sci Rep 8: 622, 2018.

24. Cao J and Zhang S: A Bayesian extension of the hypergeometric test for functional enrichment analysis. Biometrics 70: 84-94, 2014.

25. Huang da W, Sherman BT and Lempicki RA: Systematic and integrative analysis of large gene lists using DAVID bioinformatics resources. Nat Protoc 4: 44-57, 2009.

26. Lu X, Yang Y, Wu F, Gao M, Xu Y, Zhang Y, Yao Y, Du X, $\mathrm{Li} \mathrm{C}, \mathrm{Wu} \mathrm{L}$, et al: Discriminative analysis of schizophrenia using support vector machine and recursive feature elimination on structural MRI images. Medicine (Baltimore) 95: e3973, 2016.

27. Deist TM, Dankers FJWM, Valdes G, Wijsman R, Hsu IC, Oberije C, Lustberg T, van Soest J, Hoebers F, Jochems A, et al: Machine learning algorithms for outcome prediction in (chemo) radiotherapy: An empirical comparison of classifiers. Med Phys 45: 3449-3459, 2018.

28. Wang Q and Liu X: Screening of feature genes in distinguishing different types of breast cancer using support vector machine. Onco Targets Ther 8: 2311-2317, 2015.

29. Robin X, Turck N, Hainard A, Tiberti N, Lisacek F, Sanchez JC and Müller M: pROC: An open-source package for $\mathrm{R}$ and $\mathrm{S}+$ to analyze and compare ROC curves. BMC Bioinformatics 12: 77, 2011.

30. Goyette J and Geczy CL: Inflammation-associated S100 proteins: New mechanisms that regulate function. Amino Acids 41: 821-842, 2011.

31. Fontaine M, Pachot A, Larue A, Mougin B, Landelle C, Venet F, Allombert C, Cazalis MA, Monneret G and Lepape A: Delayed increase of S100A9 messenger RNA predicts hospital-acquired infection after septic shock. Crit Care Med 39: 2684-2690, 2011.

32. Heinemann AS, Pirr S, Fehlhaber B, Mellinger L, Burgmann J, Busse M, Ginzel M, Friesenhagen J, von Köckritz-Blickwede M, Ulas T, et al: In neonates S100A8/S100A9 alarmins prevent the expansion of a specific inflammatory monocyte population promoting septic shock. FASEB J 31: 1153-1164, 2017.

33. Pena OM, Hancock DG, Lyle NH, Linder A, Russell JA, Xia J, Fjell CD, Boyd JH and Hancock RE: An endotoxin tolerance signature predicts sepsis and organ dysfunction at initial clinical presentation. EBioMedicine 1: 64-71, 2014.

34. Fontaine M, Planel S, Peronnet E, Turrel-Davin F, Piriou V, Pachot A, Monneret G, Lepape A and Venet F: S100A8/A9 mRNA induction in an ex vivo model of endotoxin tolerance: Roles of IL-10 and IFN $\gamma$. PLoS One 9: e100909, 2014.

35. Venancio TM and Aravind L: CYSTM, a novel cysteine-rich transmembrane module with a role in stress tolerance across eukaryotes. Bioinformatics 26: 149-152, 2010.

36. Mastrokolias A, Ariyurek Y, Goeman JJ, van Duijn E, Roos RA, van der Mast RC, van Ommen GB, den Dunnen JT, 't Hoen PA and van Roon-Mom WM: Huntington's disease biomarker progression profile identified by transcriptome sequencing in peripheral blood. Eur J Hum Genet 23: 1349-1356, 2015.

37. Amir Shaghaghi M, Murphy B and Eck P: The SLC2A14 gene: Genomic locus, tissue expression, splice variants, and subcellular localization of the protein. Biochem Cell Biol 94: 331-335, 2016.

38. Amir Shaghaghi M, Zhouyao H, Tu H, El-Gabalawy H, Crow GH, Levine M, Bernstein CN and Eck P: The SLC2A14 gene, encoding the novel glucose/dehydroascorbate transporter GLUT14, is associated with inflammatory bowel disease. Am J Clin Nutr 106: 1508-1513, 2017.

39. Wang W, Yu JT, Zhang W, Cui WZ, Wu ZC, Zhang Q and Tan L: Genetic association of SLC2A14 polymorphism with Alzheimer's disease in a Han Chinese population. J Mol Neurosci 47: 481-484, 2012.

40. Kirchhof MG, Chau LA, Lemke CD, Vardhana S, Darlington PJ, Márquez ME, Taylor R, Rizkalla K, Blanca I, Dustin ML and Madrenas J: Modulation of T cell activation by stomatin-like protein 2. J Immunol 181: 1927-1936, 2008. 
41. Li Y, Li Y, Bai Z, Pan J, Wang J and Fang F: Identification of potential transcriptomic markers in developing pediatric sepsis: A weighted gene co-expression network analysis and a case-control validation study. J Transl Med 15: 254, 2017.

42. Roosild TP and Castronovo S: Active site conformational dynamics in human uridine phosphorylase 1 . PLoS One 5: e12741, 2010

43. Evaldsson C, Ryden I and Uppugunduri S: Anti-inflammatory effects of exogenous uridine in an animal model of lung inflammation. Int Immunopharmacol 7: 1025-1032, 2007.

44. Yamamoto K, Yuasa K, Miyagoe Y, Hosaka Y, Tsukita K, Yamamoto H, Nabeshima YI and Takeda S: Immune response to adenovirus-delivered antigens upregulates utrophin and results in mitigation of muscle pathology in mdx mice. Hum Gene Ther 11: 669-680, 2000.
45. Waheed I, Gilbert R, Nalbantoglu J, Guibinga GH, Petrof BJ and Karpati G: Factors associated with induced chronic inflammation in mdx skeletal muscle cause posttranslational stabilization and augmentation of extrasynaptic sarcolemmal utrophin. Hum Gene Ther 16: 489-501, 2005.

(i) $($ This work is licensed under a Creative Commons Attribution-NonCommercial-NoDerivatives 4.0 International (CC BY-NC-ND 4.0) License. 